\title{
Algorithms of the information stimulation system of Russian citizens' socio-optimal actions
}

\author{
M.I. Geraskin ${ }^{1}$ \\ ${ }^{1}$ Samara National Research University, 34 Moskovskoe Shosse, 443086, Samara, Russia
}

\begin{abstract}
The problem of the development of the state information stimulation system of Russian citizens' socio-optimal actions is considered according to the optimum of collective utility function as criterion. Conceptual model of the system is formed according to the conditions of individual rationality, Pareto efficiency, nonmanipulability and dynamic quasi-optimality. The algorithms of the information system are developed such as a process of step-by-step approximations. The system's criterion on each approximation does not decrease and the constraint on the stimulation fund is fulfilled.
\end{abstract}

Keywords: information system; distribution incentives mechanism; additive collective utility function; stimulation system; nonmanipulability; quasi-optimality

\section{Introduction}

In a transitional economy [1,2] trends of individual rationality are growing in the society. To overcome this trends the state develops the moral improving programs [3,4]. In this case, the purpose of the state is the social effect of citizens' acts, performing on the basis of maximization of collective but not individual utility function, hereinafter referred to as socio-optimal actions. Achieving this purpose requires the involvement in socially useful activity of large group of the population and personified registration of socio-optimal actions. It needs to organize the state information system, based on the information resources of currently working in Russia programs [5-7].

The concept of socio-optimal actions' stimulation provides for the establishment of information system of personified registration of the actions of citizens (hereinafter, agents). The system also includes the distribution of state stimulation fund in the form of incentives between agents according to certain mechanisms. The dynamics of the system is a two-period. In the first period (registration period) performed socio-optimal actions are recorded, and in the end of this period the stimulation fund is distributed. In the next period (period of stimulation) the earlier distributed incentives are used.

In addition to the utilitarian stimulating function information system also solves the problem of the formation of the agent's status in the hierarchy of the citizens, used for non-material motivation. On a longer time horizon, the state's social priorities could changed by varying the attributes of socio-optimal actions and their monetary valuation can be varied as a result of inflation. Therefore, to comparability of agents' statuses the system accumulates not only incentives as the current cash equivalent of social activity, but also agents' rating in comparable dimension.

The object of stimulation is socio-optimal actions of citizens, that is, actions that correspond to certain attributes. The actions should maximize collective utility function without increasing the individual utility function. Therefore, the attributes correspond to the terms of gratuitousness, public utility and unconnectedness with professional activities of citizens. Consequently, sociooptimal actions do not require special qualification, whereby the stimulation object's dimension is duration of action excluding the content of the action. The subject of stimulation is citizen, performing a socio-optimal action in certain period. The apparatus of stimulation is the state represented by certain ministries (departments).

\section{The object of the study}

The system under consideration is constructed outside epy architecture of market relations. Therefore, formed by market equilibrium relationship between the socio-optimal actions and incentives as their monetary valuation does not exist. For this reason, in this system it is possible some disproportions. Firstly, dynamic inconsistencies between the stimulation fund and the cash equivalent of the socio-optimal actions leads to a deficit or proficit of the fund; the deficit will not allow; the proficit expresses disinterest of citizens in stimulation. Secondly, the impossibility of compliance control between registered in the information system and the actual socio-optimal actions leads to inaccurate registration (overcharged) information. Therefore, for system's equilibrium incentive distribution mechanism must meet the following conditions: 1) individual rationality, in which the agents' utilities with the incentives are not lower than any alternative, that is, agents are interested in stimulation; 2) Pareto efficiency, which means that stimulation fund if distributed fully between agents, that is, there is no deficit and proficit; 3 ) nonmanipulability (compatible with incentives), in which each agent reported accurate information about its action according to criterion of individual rationality; 4) optimal distribution according to criterion of collective (additive) utility function. Thus, the object of study is the state information system providing equilibrium humanitarian goals of the state, the tools of their realization in the form of incentive distribution mechanism, as well as socio-optimal actions performed by citizens. 


\section{Methods}

The investigations of stimulation systems and distribution mechanisms produce the following mechanisms corresponding the individual rationality. Competitive mechanism is developed with noncooperative [8] and cooperative [9] behavior of agents, its Pareto efficiency and optimality according to additive utility function criterion are proved. The step-by-step resource distribution mechanism (SRDM) is obtained [10], for which proved [11] that nonmanipulability and Pareto efficiency simultaneously only for SRDM; also SRDM, as shown in [12], is equivalent to mechanisms of direct and reverse priorities. It was shown [13] that unique SRDM exists, in which the incentive is distributed [14] as minimum of agent's information and the average undistributed rest of incentives. The approach to the distribution based on the penalty and incentive functions [15] showed the Pareto efficiency and optimality according to additive utility function criterion for compensatory mechanisms; according to a compensatory mechanism incentives are equal to agents' costs. Thus, only SRDM satisfies [16] all above conditions. Since SRDM implies consistent registration of agents' actions and further distribution of the incentives, it is impossible to use in the system, where actions perform independently and record simultaneously. Therefore, the development of adaptive distribution algorithm, satisfying the individual rationality, Pareto efficiency, nonmanipulability and additive optimality is important.

The model of information system of stimulation is considered. We introduce the following sets. The set of socio-optimal actions attributes

$$
Z=\left\{z_{i}, i=1, \ldots, I\right\}
$$

defines the attributes of action to be stimulating; index $I$ is the number of the types of actions. The set of agents

$$
K(t)=\{1, \ldots, n(t)\}
$$

includes citizens, performing in the period $t$ actions corresponding $Z$; index $n(t)$ is the number of agents. The vector of sociooptimal actions

$$
A(Z, t)=\left\{a_{k}(Z, t), k \in K\right\}
$$

includes quantitative estimates of $k$-th agent's actions corresponding $Z$ in $t$-th period in terms of time taken to perform these actions. The vector $A(Z, t)$ is contained in allowable set

$$
\bar{A}=\left\{a_{k} \in\left[0, a^{\mathrm{max}}\right], a^{\mathrm{max}}>0, k \in K\right\},
$$

where the symbol $a^{\max }$ denotes the upper limit of agents' disposable time. For example, the vector $Z$ may include attributes such as $z_{1}=$ «carrying out socially useful activities», $z_{2}=$ «provision of free services to the citizen»; the components of the vector $A(Z, t)$ express the time registered in the $t$-th period.

The stimulation fund in the $t$-th period is

$$
F(t) \in\left(0, F^{\operatorname{mx}}\right], F^{\max }>0 .
$$

Then we omit the index $t$, assuming that all the parameters of the model correspond to a specific period.

We introduce the dimensionless registration function of socio-optimal actions

$$
u_{k}=\psi\left(a_{k}\right), k \in K,
$$

which the score value $u$ corresponds to a time value $a$. Thus, the vector of action $A$ corresponds to the vector of scores

$$
U=\left\{u_{k}, k \in K\right\} \in \bar{U},
$$

where $\bar{U}$ - allowable set of scores. Let a function $\psi(\bullet)$ is continuously differentiable, satisfies the conditions of saturation and set is defined $\bar{U}$ as follows:

$$
u_{k}=\psi\left(a_{k}\right): \psi_{a}^{\prime}(\bullet)>0, \psi_{a}^{\prime \prime}(\bullet)<0, k \in K, \bar{U}=\left\{u_{k} \in\left[0, u^{\max }\right], u^{\max }>0, k \in K\right\}, u^{\max }=\psi\left(a^{\max }\right)>0 .
$$

We introduce the social utility function of agent $f(a, x)$ as the sum of the individual utility function and distributed incentive

$$
f(a, x) f_{k}\left(a_{k}, x_{k}\right)=f_{k}^{0}\left(a_{k}\right)+x_{k}\left(u_{k}\right), k \in K,
$$

where $f^{0}(\bullet)$ - individual utility function; $x(u)$ - distributed incentive.

For the function (2) property of individual rationality is defined as: the social utility function of agent performing the action $a$ must be no less than the individual utility function when such action does not perform $(a=0)$, that is,

$$
f_{k}\left(a_{k}, x_{k}\right) \geq f_{k}^{0}(0), k \in K \text {. }
$$

Therefore, incentives at the individual rationality must satisfy to

$$
x_{k}\left(u_{k}\right) \geq f_{k}^{\mathrm{min}}, k \in K
$$

where

$$
f_{k}^{\text {min }}=f_{k}^{0}(0)-f_{k}^{0}\left(a_{k}\right) \geq 0
$$

characterize the loss of individual utility when making the socio-optimal action. Agents can't have individual utility losses when making socio-optimal action, for example, performing it in their spare time from gainful activity. Therefore, guaranteed estimate of these losses, the same for all agents, is

$$
f^{\min }=\arg \max _{k \in K} f_{k}^{\min } \text {. }
$$

We introduce the stimulation function $x=\varphi(u)$, which the incentive in cash corresponds to the score $u$. Let the function $\varphi(\bullet)$ is continuously differentiable, satisfies unsaturation condition on $u$ and saturation condition on the sum of $u$, individual rationality and Pareto efficiency: 


$$
x_{k}=\varphi\left(u_{k}\right): \varphi_{u_{k}}^{\prime}(\bullet)>0, \varphi_{\sum_{k \in K} u_{k}}^{\prime \prime}(\bullet)<0, x_{k}\left(u_{k}\right) \geq f^{\text {min }} \geq 0, \sum_{k \in K} x_{k}\left(u_{k}\right)=F, k \in K .
$$

Generally formulas (1) - (3) are the stimulation system model $S$ that represents the list of agents' set, agents' scores set and agents' utility functions set:

$$
S=\left\langle K, \psi_{k}\left(a_{k}\right), f_{k}\left(a_{k}, x_{k}\left(u_{k}\right)\right), k \in K\right\rangle .
$$

We introduce the additive criterion of social efficiency of the stimulation system as the sum of the socio-optimal actions of agents in the $t$-th period

$$
E(S)=\sum_{k \in K} a_{k}(S)
$$

Definition: a nonmanipulability - is a property of the system $S$ having the Nash equilibrium, that is, a state in which there is no agent, for which the social utility function for some action vector $\widetilde{A} \in \bar{A}$ is greater than the equilibrium Nash vector $A^{N} \in \bar{A}:$

$$
\bar{\exists} k \in K: f_{k}\left(x_{k}(\tilde{A})\right)>f_{k}\left(x_{k}\left(A^{N}\right)\right), \tilde{A}=\left(\tilde{a}_{k}, a_{-k}\right), \tilde{A}, A^{N} \in \bar{A},
$$

where symbol «-k» denotes environing, i.e. agents other than $k$-th.

We define the set of allowable stimulation systems $\bar{S}$ based on the conditions of Pareto efficiency and individual rationality (3), nonmanipulability (6) in the form of:

$$
\bar{S}=\left\{S: x_{k} \geq f^{\min }, \sum_{k \in K} x_{k} \leq F, \bar{\exists} k \in K: f_{k}\left(x_{k}(\tilde{U})\right)>f_{k}\left(x_{k}(U)\right)\right\} .
$$

The problem of selecting the model $S$ from the set $\bar{S}$ is considered as the optimal control problem

$$
S^{*}=\arg \max _{S \in \bar{S}} E(S) \text {. }
$$

The control parameters in the problem $(8)$ is a functions $\psi(\bullet), \varphi(\bullet)$. So the criterion and constraints in problem (8) implicitly depend on the control parameters, and the problem (8) does not, in general, the analytical solution. In these cases, the approximate methods are used [17-21] for solution of optimal control problem, and the resulting solution was called quasioptimal [22].

Definition: a model of stimulation system $S$ is called a dynamic quasi-optimal if

$$
E_{t}^{\prime}(t) \geq 0 \forall S \in \bar{S}
$$

The dynamic quasi-optimality means that in $t$-th period additive criterion system (5) is no less the value at $(t-1)$-th period when the constraints (7). Therefore, the condition (9) defines the process of step-by-step approximations for system (4), on each of which the criterion (5) does not decrease. We pose the problem of developing an algorithm that implements the model (4) as a dynamic quasi-optimal. Subsequently, the problem of developing an algorithm that implements the model (4) as a dynamic quasi-optimal, is considered.

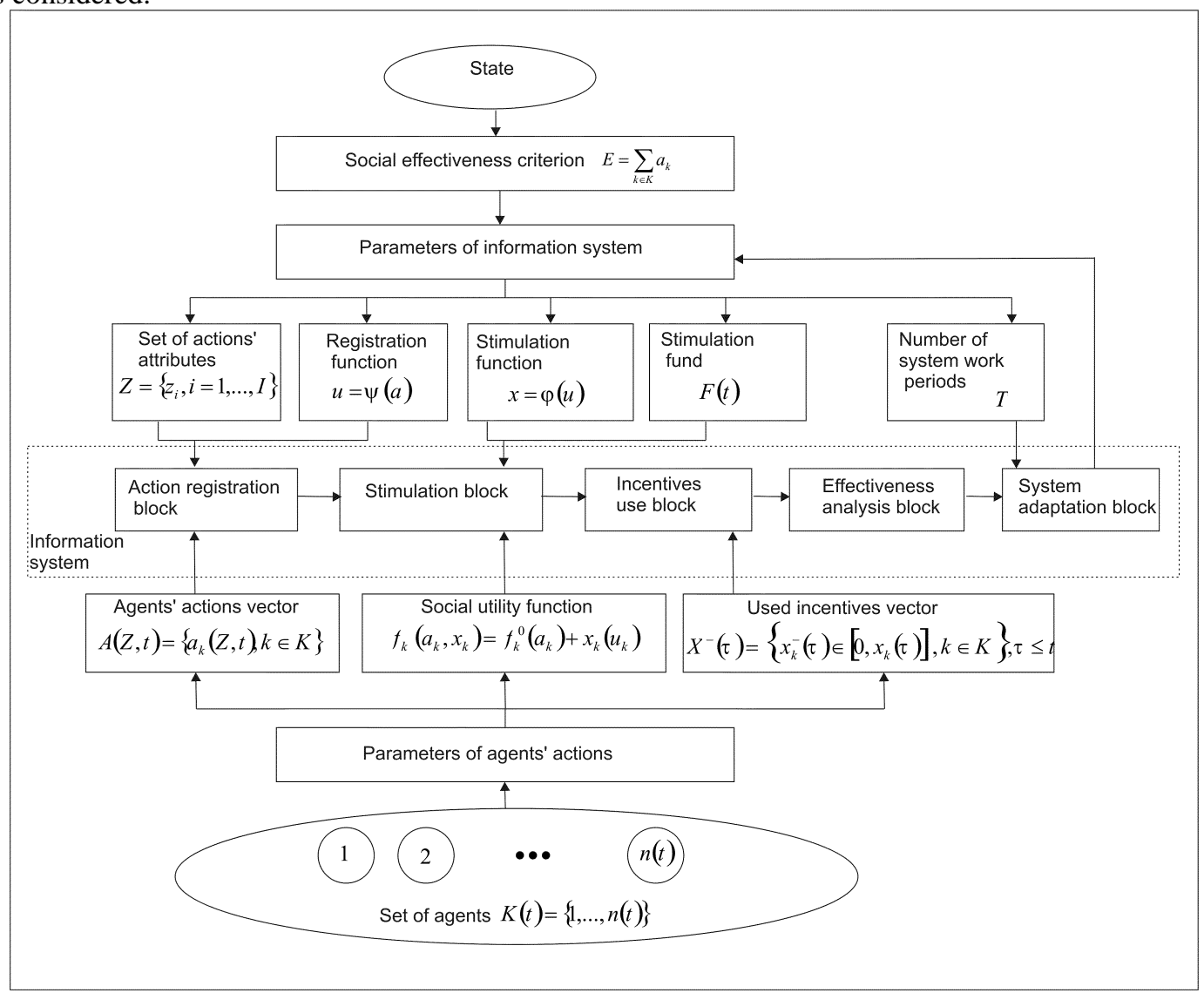

Fig. 1. Conceptual model of information system. 


\section{Results and discussion}

Conceptually, the projected information system (Fig. 1) implements the processes of interaction between state and citizens based on the goal of maximizing the total number of socio-optimal actions [23,24].

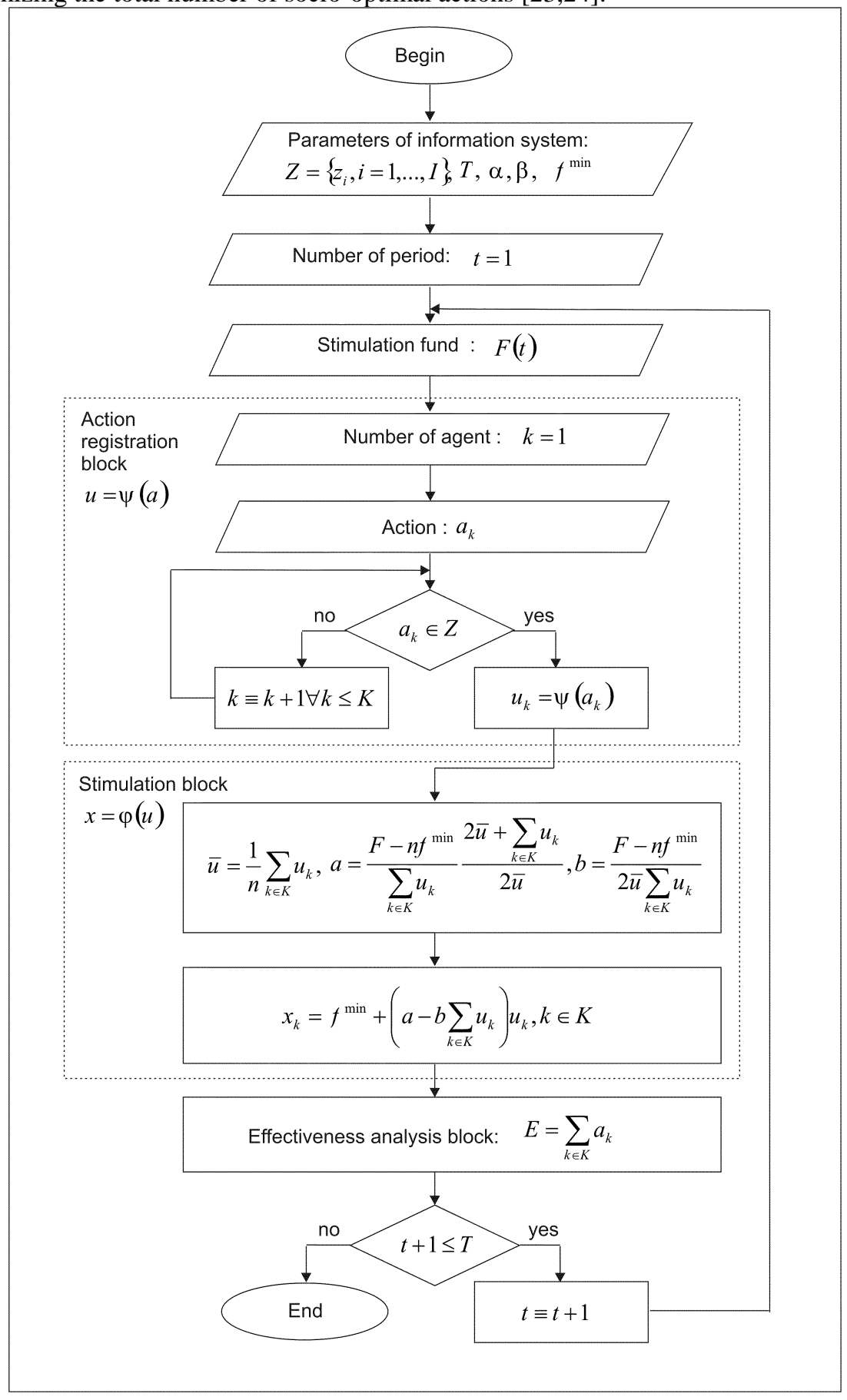

Fig. 2. Algorithm of one-period cycle of the information system.

The state defines such parameters of information system as a set of actions' attributes, the form of registration function, the form of stimulation function, the stimulation fund and the number of system work periods. The agents' actions are parameterized via the vector of action, a set of social utility functions and the vector of used incentives. The information system is the infrastructure of the state and citizens, consisting of five blocks. The action registration block identifies actions in the score values. The stimulation block is designed to distribute the fund, depending on the vector of score ratings. The incentives use block implements the functions of analyzing the dynamics of accrued and used incentives, as well as control the deficit (proficit) of the stimulation fund. The effectiveness analysis block controls the dynamics of change in the social effectiveness criterion of system test on the selected time interval of up to the maximum number of work periods. The system parameters adaptation block implements a process of successive approximations for quasi-optimality.

A degree registration function $\psi(\bullet)$ satisfying the conditions (1) is considered in the form

$$
\psi\left(a_{k}\right)=\alpha a_{k}^{\beta}, \alpha \in\left(0, \alpha^{\operatorname{mx}}\right], \beta \in\left(0, \beta^{\max }\right], \beta^{\max } \in(0,1], k \in K,
$$

where $\alpha, \beta$ are constant coefficients. 
We introduce the stimulation function $\varphi(\bullet)$ relating to the class of direct compensatory priority functions [25]:

$$
\varphi\left(u_{k}\right)=f^{\mathrm{min}}+\left(a-b \sum_{k \in K} u_{k}\right) u_{k}, k \in K,
$$

where $a, b$ are constant coefficients, $a, b>0$, are selected to satisfy $\varphi(\bullet)$ the conditions (3). Substantially compensatory function (11) defines guaranteed incentive $f^{\text {min }}$ of non-zero agent's action on the principle of "any socially useful action is rewarded", beyond which the incentive is distributed in proportion to the score $u$ in accordance with the "cost" $p$

$$
p=a-b \sum_{k \in K} u_{k} \text {, }
$$

decreasing with increasing the total number of agents.

It can be shown that if the coefficients $a, b$ are defined by the formulas

$$
a=\frac{F-n f^{\min }}{\sum_{k \in K} u_{k}} \frac{2 \bar{u}+\sum_{k \in K} u_{k}}{2 \bar{u}}, b=\frac{F-n f^{\min }}{2 \bar{u} \sum_{k \in K} u_{k}}, \bar{u}=\frac{1}{n} \sum_{k \in K} u_{k},
$$

the system $S$ provides firstly Pareto efficient distributed of stimulation fund, that is, the full distribution without deficit or proficit and, secondly, the agents are not interested in the overstated information about performed actions, that is, the system is nonmanipulable.

The algorithm for static (one-period) cycle of the information system in shown in Fig. 2. The one-period cycle does not include the incentives use phase, since the distribution of incentives is possible only after the registration of all actions of all agents in that period. The one-period cycle information system algorithm includes the action registration block, the stimulation block and the effectiveness analysis block.

The one-period cycle does not allow to achieve the stimulation system optimality according to criterion (5), in particular, to adapt the parameters of the blocks « $u=\psi(a) »,\langle x=\varphi(u) »$ so that the quasi-optimality condition (9) is performed in dynamics. In addition, the one-period cycle is not taken into account the dynamics of agents' number increasing and the dynamics of actions number, resulting in incentives inflation in case of constant stimulation fund, which may result in loss of efficiency. Also, in the one-period cycle the used incentives dynamics is not coordinated with the dynamics of stimulation fund, which could lead to its deficit or proficit, and causes a reduction in efficiency.

The dynamic (multi-period) algorithm of the information system is considered. We introduce the following parameters of the dynamics of the system in the period $\tau \in(0, t]$ : the accrued incentives vector is $X(\tau)$, the components of which are defined by (11), the used incentives vector is $X^{-}(\tau)$, the used stimulation fund is $F^{-}(\tau)$, the unused incentives residues vector at the end of the $t$-th period is $R(t)$, the total unused residue of incentives at the end of $t$-th period is $R \Sigma(t)$, the unused residue of stimulation fund at the end of $t$-th period is $\Phi$. These parameters are determined by the following formulas:

$$
\begin{aligned}
& X(\tau)=\left\{x_{k}\left(u_{k}(\tau)\right), k \in K\right\} ; X^{-}(\tau)=\left\{x_{k}^{-}(\tau) \in\left[0, x_{k}(\tau)\right], k \in K\right\} ; F^{-}(\tau)=\sum_{k \in K} x_{k}^{-}(\tau), \tau \in[2, t] ; \\
& R(t)=\left\{r_{k}(t)=\sum_{\tau=1}^{t} x_{k}(\tau)-\sum_{\tau=2}^{t} x_{k}^{-}(\tau), k \in K\right\} ; R \Sigma(t)=\sum_{k \in K} r_{k}(t) ; \Phi(t)=\sum_{\tau=1}^{t} F(\tau)-\sum_{\tau=2}^{t} F^{-}(\tau),
\end{aligned}
$$

where $x^{-}(\tau)$ is incentive used by $k$-th agent in the period $\tau \leq t$.

In the case of full distribution of stimulation fund (Pareto efficiency), it can be shown that if the following conditions are met in the $t$-th period

$$
\Phi(t)=R \Sigma(t) \geq 0, E(t)-E(t-1)>0, t \in(2, T],
$$

the information system is quasi-optimal. Violation of the conditions (13) indicates the non-optimality of the system (4) in the $t$ the period, that is, the need to adapt such system parameters as registration function coefficients $\alpha, \beta$ and fund $F$.

The algorithm of information system multi-period cycle is shown in Fig. 3. Blocks, detailed in the one-period cycle (Fig. 2), are shown in general. The incentives use block implements formulas (13), (14). The system parameters adaptation block is based on the analysis of conditions (15), the variations of system parameters are defined by the formulas:

$$
\Delta \alpha \in\left[0, \alpha^{\max }-\alpha(t-1)\right], \Delta \beta \in\left[0, \beta^{\max }-\beta(t-1)\right], \Delta F \in\left[0, \min \left\{F^{\max }-F(t-1),|R \Sigma(t)|\right\}\right] .
$$

The algorithm provides a process of successive approximations, when the constraints are complied, resulting in the system to quasi-optimality state.

Simulation of the stimulation impact on the behavior of the population was carried out by changing the skewness and kurtosis of probability density function of the normal distribution

$$
f(a)=\frac{1}{\sigma \sqrt{2 \pi}} e^{-\frac{w\left(a-\bar{a}^{l}\right)^{2}}{2 \sigma^{2}}} .
$$

where $\bar{a}, \sigma$ - mathematical expectation and mean-square deviation of initial distribution; $l$ - skewness coefficient $(l<1-1$ left skewness, $l>1$ - right asymmetry) compared with a normal distribution $(l=1)$; $w$ - kurtosis coefficient ( $w<1$ - a more uniform distribution, $w>1$ - less uniform distribution) as compared with the normal distribution $(w=1)$. 


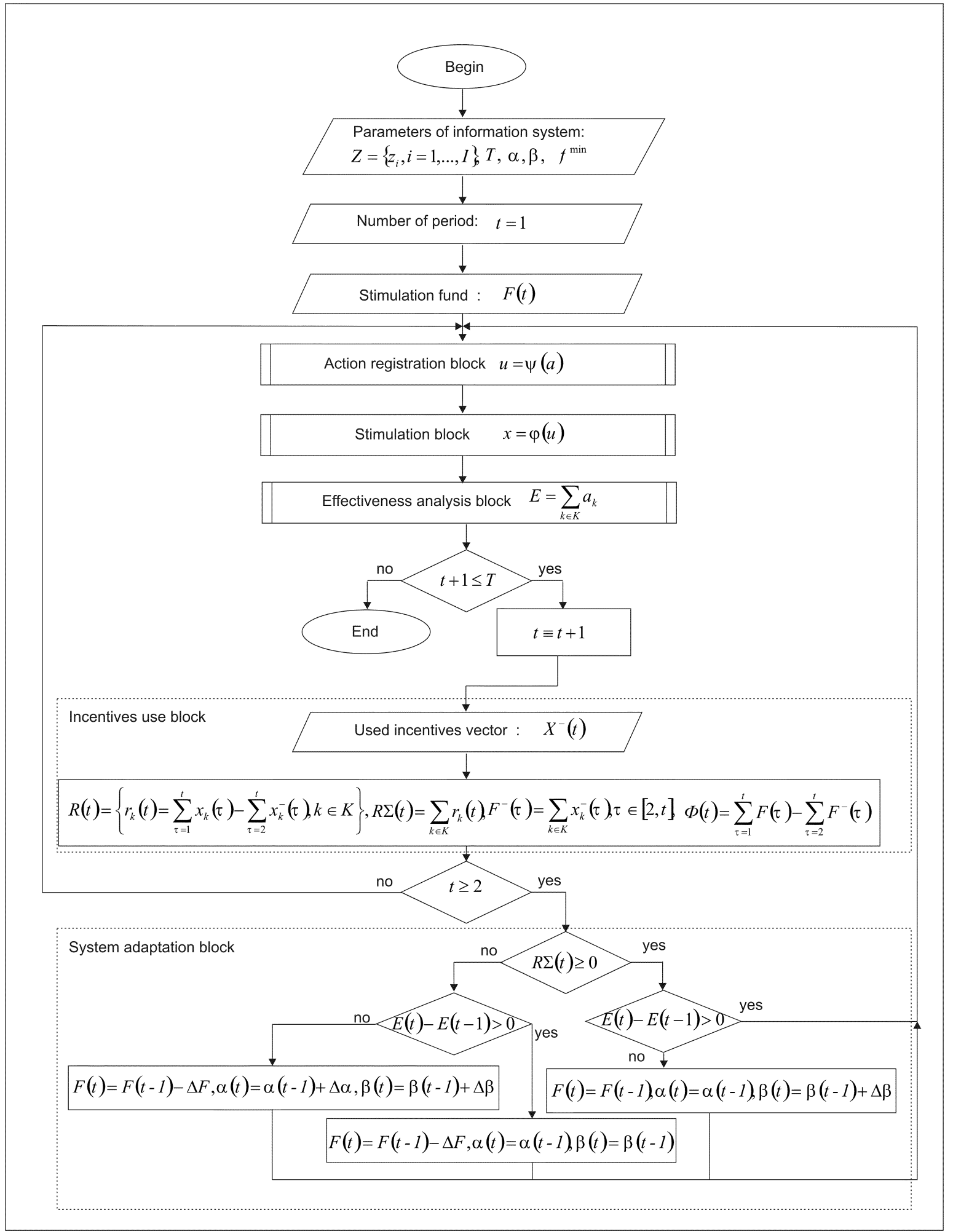

Fig. 3. Algorithm of multi-period cycle of the information system.

The simulation of the distribution of agents in the stimulation process was based on the following hypothesis: registration function coefficient $\beta$ growth leads to right skewness, i.e., to increase of the expectation of the distribution of the agents' set, compared with the median of range [0, $\left.a^{\max }\right]$; coefficient $\alpha$ growth leads to decrease in kurtosis, to increase the variance of the distribution of the agents' set in comparison with the initial value. The agents' number changing in the stimulation process was not more than $20 \%$ of the initial number of citizens, which was led to constraint on the coefficients of skewness and kurtosis $l \in[1,1.4], w \in[0.7,1]$. The simulation of information system was carried out for the following initial data: $a^{\text {max }}=8$ hours, $\bar{a}=4$ hours, $\sigma=1.293$ hours, $f^{\text {min }}=1$ thousand rubles, $n=9727$ thousand. The initial fund value was established $F=1000000$ thousand rubles, the initial registration function coefficients $\alpha=18,95, \beta=0,8$ were chosen from the condition $u^{\max }=100$; the value of the used incentives coefficient, i.e. share of the used incentives to stimulation fund, was taken $\Delta X=0.8$.

Three scripts of information system dynamics were considered: the first script (Fig. 4.) - the growth $\beta$ when $\alpha=$ const, $F=$ const, $\Delta X=$ const led to a right skewness; the second script (Fig. 5.) - the growth $\alpha$ when 
$\beta=$ const, $F=$ const, $\Delta X=$ const led to the reduction of kurtosis; the third script (Fig. 6). - the growth $\beta$ when $\alpha=$ const,$F=$ const resulted in right skewness, and with the increase $\Delta X$ stimulation fund $F$ reduced on the relative value $\Delta F$.

The dynamics of the first script is shown (Fig. 4) in the periods $t=1, \ldots, 7$, for which the coefficient $\beta$ was varied in the range $[0.8,0.92]$, which led to increase in the coefficient $l \in[1,1.4]$. As a result, the maximum average value of system efficiency reached $E_{a v}=5.68$, the average stimulation fund residue $R_{a v}$. increased to 200, the average score $u_{\mathrm{av}}$. increased to 111 , the score price decreased to $p=1.28$.

The dynamics of the second script (Fig. 5) for changing the coefficient $\alpha$ in the range [18.95,19.25] led to decrease in the coefficient $w \in[0.7,1]$. As a result, the following figures were found: $E_{a v}=4.76, R_{a v .}=121, u_{\mathrm{av} .}=57, p=1.48$.

The dynamics of the third script (Fig. 6) repeated the first script of the coefficient $\beta$ change in the range [0.8,0.92], which led to growth of coefficient $l \in[1,1.4]$. However, at $t=1, \ldots, 6$ stimulation fund has been fixed $(\Delta \mathrm{F}=0)$ and the follow used incentives coefficient has been set: $\Delta X=0.8$ at $t=1,2, \Delta X=1.1$ at $t=3, \ldots, 7$. As a result, at $t=6$ value $R_{a v}=0$ was reached, that led to the need at $t=7$ to finish stimulation $(\Delta \mathrm{F}=1)$; at $t=7$ the following figures were obtained:. $E_{a v}=4.91, R_{a v}=0 u_{\mathrm{av} .}=111, p=0$.

The simulation showed the following results: 1) the average efficiency of the stimulation system is more sensitive to a change in the registration function coefficient $\beta$ by right skewness of the distribution of the agents' set than to a change in the coefficient $\alpha$ by reducing the kurtosis of the distribution, because of in the first case, the number of agents decreases, while in the second increases; 2) stimulation system is non-manipulable, because of the score price decreases with increasing registration function coefficients; 3) stimulation fund deficit occurs when an excessive use of incentives, and deficit is compensated by fund decrease in the subsequent period.

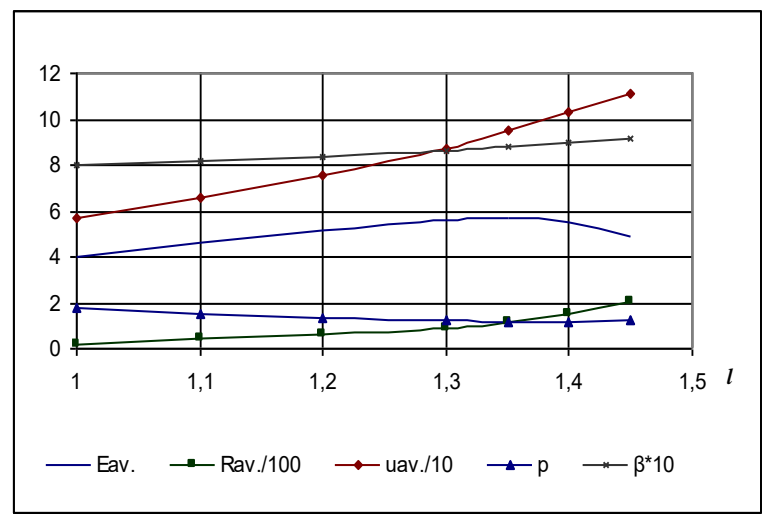

Fig. 4. The dynamics of the information system first script, the values of the coefficient $l$ correspond to the periods $t=1, \ldots, 7$.

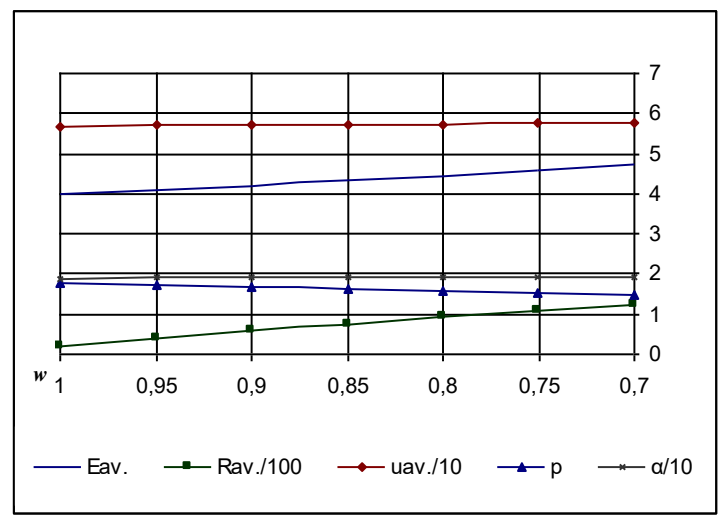

Fig. 5. The dynamics of the information system second script, the values of the coefficient $w$ correspond to the periods $t=1, \ldots, 7$.

\section{Conclusion}

The problem of information support of the state strategy of strengthening morality in society was considered. The information system of stimulation of citizens' actions was developed based on collective utility function maximizing. In the article the following results were obtained. The conceptual model of information system was formed on the base of individual rationality, Pareto efficiency, non-manipulablity and dynamic quasi-optimality. The model includes the action registration block, the stimulation block, the incentives use block, the effectiveness analysis block and the system parameters adaptation block.

The specific form of the compensatory stimulation function of the direct priorities class was proposed. In this function, the incentive consists of a guaranteed minimum and proportional to agent' score "premium". The score price decreases with the growth of the total number of agents' scores. This stimulation function implements the mechanism similar to the mechanism of oligopoly market equilibrium [26], but, unlike that, for certain function coefficients the agents' actions vector is Pareto efficient. Unlike the compensatory mechanism [27,28] proposed stimulation function ensures Nash equilibrium actions vector in such case than the stimulation fund does not depend on the actions vector. Thus, formed stimulation system satisfies the conditions of Pareto efficiency and compatibility with incentives. 


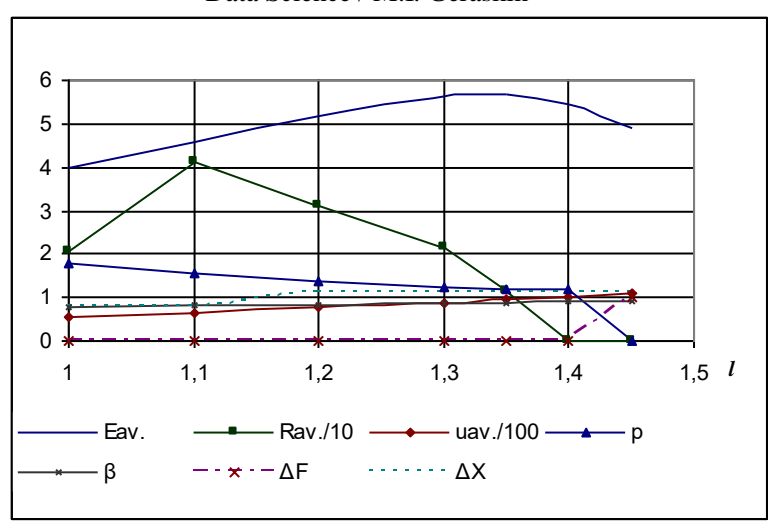

Fig.6. The dynamics of the information system third script, the values of the coefficient $l$ correspond to the periods $t=1, \ldots, 7$.

The dynamic algorithm for information system was developed as a multi-period cycle, which includes a one-period cycle of the actions registration and the stimulation fund distribution. The algorithm implements a process of step-by-step approximations with constraints, which result in a quasi-optimality system state. In this case, the system criterion does not decrease, and condition of stimulation fund sufficiency is fulfilled.

\section{References}

[1] Roland G. Transition and Economics. Politics, Markets, and Firms. Cambridge: MIT Press, 2000; 840 p.

[2] Braguinsky S, Yavlinsky G. Incentives and Institutions. Transition to a Market Economy in Russia. Princeton. NJ.: Princeton University Press, 2000; 420 p.

[3] RF Government Decree of December 302015 N 1493 "On State program" Patriotic Education of Citizens of the Russian Federation for 2016 - 2020".

[4] RF Government Decree of December 272012 N 2567-r "On the state program of the Russian Federation" Development of Culture and Tourism "2013 2020".

[5] RF Government Decree of April 152014 N 313 (as amended on 10.21.2016.) "On approval of the Russian Federation, the state program" Information Society (2011 - 2020".

[6] RF Government Decree of December 272012 N 1406 (as amended on 12.25.2015.) "On the federal target program" Development of the Russian judicial system for 2013 - 2020".

[7] RF Government Decree of April 152014 N 320 "On approval of the state program of the Russian Federation" Public Financial Management and regulation of financial markets".

[8] Burkov VN, Danev B, Enaleev AK, Nanev TB, Podvalny LD, Yusupov BS. Competitive mechanisms in problems of distribution of scarce resources. Avtomatika i telemekhanika 1988; 11: 142-53.

[9] Burkov VN, Enaleev AK, Kalenchuk VF. Coalition with the competitive mechanism of resource distribution. Avtomatika i telemekhanika 1989; 12: 81-90.

[10] Burkov VN, Enaleev AK, Lavrov YG. Synthesis of optimal planning and incentive mechanisms in the active system. Avtomatika i telemekhanika 1992; 10: $113-120$.

[11] Burkov VN, Iskakov MB, Korgin NA. Application of generalized median schemes for the construction of non-manipulable mechanism multicriterion active expertise. Automation and Remote Control 2010; 71(8): 1681-1694.

[12] Korgin NA. Equivalence of non-manipulable and non-anonymous priority resource distribution mechanisms. Upravleniye bol'shimi sistemami 2009; 26(1): 319-347.

[13] Burkov VN, Gorgidze II, Novikov DA, Yusupov BS. Models and cost and revenue distribution mechanisms in the market economy. Moskva: Institut problem upravleniya 1997; $356 \mathrm{p}$.

[14] Korgin NA. Use of intersection property for analysis of feasibility of multicriteria expertise results. Automation and Remote Control 2010; 71(6): 11691183.

[15] Enaleev AK. Optimal incentive-compatible mechanisms in active systems. Automation and Remote Control 2013; 74(3): 491-505.

[16] Burkov VN, Korgin NA, Novikov DA. Problems of aggregation and decomposition mechanisms of management of organizational and technical systems. Problemy upravleniya 2016; 5: 14-23.

[17] Krylov IA, Chernousko FL. A method of successive approximations for solution optimal control problems. USSR Computational Mathematics and Mathematical Physics 1963; 2(6): 1371-1382.

[18] Gindes VB. A method of successive approximations for solution linear optimal control. USSR Computational Mathematics and Mathematical Physics 1970; 10(1): 297-307.

[19] Fedorenko RP. An approximate solution of the optimal control problems. Moskva: Nauka, 1978; 680 p.

[20] Chernous'ko FL, Kolmanovsky VB. Computational and approximate methods of optimal control. Journal of Soviet Mathematics 1979; 12 (3): 310-353.

[21] Lyubushin AA. Modifications of the method of successive approximations for solving optimal control problems. USSR Computational Mathematics and Mathematical Physics 1982; 22(1): 29-34.

[22] Aleksandrov VM, Dykhta VA. Approximate solution to the resource consumption minimization problem. I. Construction of a quasioptimal control. Journal of Applied and Industrial Mathematics 2011; 5(4): 467-477.

[23] Ivanov DU, Orlova CU, Ajupov AA, Bogatirev VD, Pavlova EV. Venture capital management technique based on real options. International Business Management 2016; 10(22): 5286-5290.

[24] Gerasimov KB, Gerasimov BN. Modeling the development of organization management system. Asian Social Science 2015; 11 (20): 82-89.

[25] Novikov D. Theory of Control in Organizations. New York: Nova Science Publishers, 2013; 341 p.

[26] Geraskin MI, Chkhartishvili AG. Structural Modeling of Oligopoly Market under the Nonlinear Functions of Demand and Agents' Costs. Automation and Remote Control 2017; 78(2): 332-348.

[27] Geraskin MI. The optimal mechanism for the distribution of the effect in an integrated strongly coupled system of anonymous agents with transferable utility. Problemy upravleniya 2017; 2: 27-41.

[28] Geraskin MI. Transferable utility distribution algorithm for multicriteria control in strongly coupled system with priorities. CEUR Workshop Proceedings 2016; 1638: 542-551. 